\title{
Custodial glide symmetry of quantum spin Hall edge modes in WTe $\mathrm{W}_{2}$ monolayer
}

\author{
Seulgi Ok, ${ }^{1}$ Lukas Muechler, ${ }^{2}$ Domenico Di Sante, ${ }^{3}$ Giorgio Sangiovanni, ${ }^{3}$ Ronny Thomale, ${ }^{3,}$ * and Titus Neupert ${ }^{1},\left.\right|^{\dagger}$ \\ ${ }^{1}$ Department of Physics, University of Zurich, Winterthurerstrasse 190, 8057 Zurich, Switzerland \\ ${ }^{2}$ Department of Chemistry, Princeton University, Princeton, New Jersey 08544, USA \\ ${ }^{3}$ Institute for Theoretical Physics and Astrophysics, University of Würzburg, Am Hubland, D-97074 Würzburg, Germany
}

\begin{abstract}
A monolayer of $\mathrm{WTe}_{2}$ has been shown to display quantum spin Hall (QSH) edge modes persisting up to $100 \mathrm{~K}$ in transport experiments. Based on density-functional theory calculations and symmetry-based model building including the role of correlations and substrate support, we develop an effective electronic model for $\mathrm{WTe}_{2}$ which fundamentally differs from other prototypical QSH settings: we find that the extraordinary robustness of quantum spin Hall edge modes in $\mathrm{WTe}_{2}$ roots in a glide symmetry due to which the topological gap opens away from high-symmetry points in momentum space. While the indirect bulk gap is much smaller, the glide symmetry implies a large direct gap of up to $1 \mathrm{eV}$ in the Brillouin zone region of the dispersing edge modes, and hence enables sharply boundary-localized QSH edge states depending on the specific boundary orientation.
\end{abstract}

Introduction - The quantum spin Hall effect (QSHE) has largely initiated the era of topological insulators [1-4] and semimetals in contemporary condensed matter research. Moving beyond its fundamental relevance as a new quantum state of matter, however, technological applications can only be brought within reach if QSHE is realized at high operating temperatures. After low temperature realizations in $\mathrm{HgTe} / \mathrm{CdTe}$ quantum wells [5-7] and InAs/GaSb heterostructures [8-10], bismuthene has set a new paradigm for hightemperature QSHE, where the bulk gap is proportional to twice the atomic spin-orbit coupling of $\mathrm{Bi}$ and thus reaches up to $0.8 \mathrm{eV}[11,12]$. In many respects, it came as a surprise when a monolayer of the dichalcogenide $\mathrm{WTe}_{2}$ as yet another material class was subsequently reported to display QSHE up to $100 \mathrm{~K}$ [13]. There is no evidence for a particularly large bulk gap in $\mathrm{WTe}_{2}$, and in view of how significantly the bulk gap in the clean limit is usually reduced to arrive at the actual gap appearing in transport data, the enormous robustness of QSHE in $\mathrm{WTe}_{2}$ poses a fundamental quest to be resolved.

$\mathrm{WTe}_{2}$ is a material whose manifold intricacies arise from its spin-orbit coupled band structure combined with the $\mathrm{W} d$ orbitals that hint at electronic correlations [13-24]. As a three-dimensional bulk material [21, 25, 26], it shows a record-high magnetoresistance of about a million percent [15]. It has further been predicted to be a Weyl semimetal with strongly Lorentz symmetry breaking type-II Weyl cones [16]. In the form of monolayers, $\mathrm{WTe}_{2}$ was already predicted to be a two-dimensional topological insulator [14, 18,-20] before it was subsequently confirmed experimentally [13, 22]. Most recently, superconductivity has been observed in a slightly doped $\mathrm{WTe}_{2}$ monolayer [23], which further stresses the potential role of electronic correlations in the compound.

In this Letter, we develop an effective low-energy electronic model for $\mathrm{WTe}_{2}$ monolayers. This is accomplished by different successive steps. First, we analyze the density functional theory description and symmetries of $\mathrm{WTe}_{2}$ in light of the existing experimental evidence, and address how the measured gapped electronic structure could be rationalized through correlations or substrate effects. Second, we distill an 8-band model for $\mathrm{WTe}_{2}$ where we identify the dominant atomic and
Rashba spin-orbit terms. As such, our effective model can be conveniently adjusted to fit monolayer $\mathrm{WTe}_{2}$, and possibly other related QSH materials, for different experimental setups. Third, we investigate the QSH edge modes in $\mathrm{WTe}_{2}$ for armchair and zigzag terminations. Due to the glide symmetry of the $\mathrm{WTe}_{2}$ monolayer, the Dirac cones shift away from highsymmetry points and, in particular for a zigzag termination, allow for the formation of a big direct gap $\sim 1 \mathrm{eV}$ protecting the QSH edge modes. Our analysis points towards a significant termination sensitivity of the edge modes, suggesting various experimental investigations motivated by our findings.

Density functional theory analysis - The lattice structure of monolayer $\mathrm{WTe}_{2}$, shown in Fig. $1 \mathrm{1}$, is composed of zig-zag chains of $\mathrm{W}$ atoms running along the $\hat{x}$ direction. The zigzag nature of the chains endows the lattice with a glide-mirror symmetry that sends $y \rightarrow-y$ combined with half a lattice translation along the chain. As a common substrate support, $\mathrm{WTe}_{2}$ can be deposited on top of bilayer graphene (Fig. 1p). From a deconstructionist perspective, monolayer $\mathrm{WTe}_{2}$ in the absence of spin-orbit coupling would be a two-dimensional topological semimetal with two gapless Dirac cones that are protected by the non-symmorphic glide-mirror symmetry. A band gap arises when spin-orbit coupling is included and gaps out the Dirac cones. As long as the non-symmorphic symmetry and time-reversal are preserved, the resulting insulator has to be topological [27]. In contrast to many other topological insulators, the Dirac cones of monolayer $\mathrm{WTe}_{2}$ are not located at high-symmetry points in momentum space, but along the glide-mirror line.

The location of the Dirac nodes off high-symmetry points is a challenge for accurate first-principle calculations of the low-energy band structure, since slight relative shifts between the energy of the valence band maximum at the $\Gamma$ point and the energy of the Dirac points severely affect the fermiology. There is ambiguous experimental evidence with regard to the nature of the electronic state. Recent angle-resolved photoemission spectroscopy (ARPES) experiments suggest the existence of a fully gapped band structure [31], while scanning tunneling microscopy (STM) experiments suggest a metallic state [32]. Density functional theory (DFT) calculations based on the generalized gradient approximation (GGA) predict a 

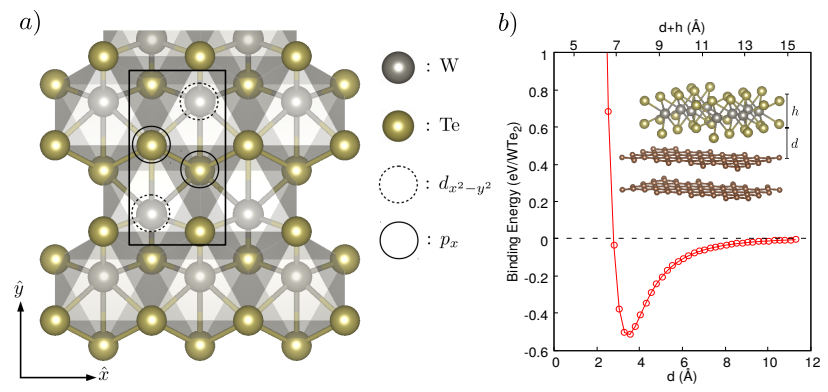

FIG. 1. a) Lattice structure and unit cell of monolayer $\mathrm{WTe}_{2}$. Dashed (solid) circles indicate Wannier functions with $d_{x^{2}-y^{2}}\left(p_{x}\right)$ symmetry contributing to the low-energy physics. The other two Te atoms in the unit cell do not play a role in the effective low-energy electronic structure. A termination parallel to the $\hat{x}(\hat{y})$ direction corresponds to the zig-zag (armchair) edge. b) Binding energy of monolayer $\mathrm{WTe}_{2}$ (height $h$ ) deposited on a $d$-distant bilayer graphene (BLG) computed via a van der Waals corrected functional.

type-II Dirac semimetal without spin-orbit coupling (SOC). The Dirac cones gap into overlapping electron and hole pockets once SOC is included, thus still rendering the resulting state metallic. It is, however, not clear whether GGA describes the electronic structure correctly. Rather, the ARPES band structures are well reproduced by HSE06 calculations of free standing monolayers [31]. This is in agreement with quantum oscillation experiments on bulk $\mathrm{WTe}_{2}$, hinting that GGA calculations fail to reproduce the electronic structure close to the Fermi level [33]. As an added complication, experiments on the monolayers likely cannot be directly compared with DFT calculations, since monolayers of $\mathrm{WTe}_{2}$ are usually realized on substrates. Even though van-der-Waals (vdw) coupled substrates might only have a moderate effect in terms of direct hybridization, the substrate could still strain the monolayer, which can induce a transition from a metallic to an insulating state within GGA calculations. Furthermore, the substrate could break the glide symmetry of the monolayer, leading to other gap opening mechanisms besides SOC.

Gap from Fock exchange - We start out by showing how the inclusion of a certain amount of exact exchange within DFT leads to a semiconducting ground state and to the opening of a positive indirect gap in monolayer $\mathrm{WTe}_{2}$, which would be one way to address the discrepancy between ARPES experiments and GGA calculations. In $\mathrm{WTe}_{2}$, the tilting of the Dirac cones is controlled by the hoppings along the chain directions and therefore a possible overestimate of these hoppings could account for the metallic band structure predicted by GGA. The inclusion of exact exchange in hybridfunctionals such as HSE06 has been shown to mitigate the effects of self-interaction errors, and therefore is expected to reverse the overlap of the electron and hole pockets along $\Gamma X$. The HSE06 band structure plotted in Fig. $2 \mathrm{a}$ indeed presents a direct gap [34]. The inset of Fig. 2a shows the influence of exact exchange on the indirect band gap, modeled by varying the fraction of exact exchange $\alpha$ [35]. The GGA functional corresponds to $\alpha=0$, while the HSE06 functional corresponds to $\alpha=0.25$. The indirect band gap $\Delta=E_{\text {con }}\left(\boldsymbol{k}_{c}\right)-E_{\text {val }}(\Gamma)$ is measured from the energy minimum of the conduction band at $\boldsymbol{k}_{c}$ relative to the valence band maximum at $\Gamma$. The indirect band gap shows a linear dependence on the amount of exact exchange and switches sign from negative to positive at $\alpha \simeq 0.2$, indicating the importance of post-GGA correlation effects in monolayers of $\mathrm{WTe}_{2}$.

Gap from the substrate - Strain induced by a substrate can also reduce the hopping strength along the chains in $\mathrm{WTe}_{2}$ and may therefore be an alternative origin for the formation of an indirect gap. In actual experimental settings, a free-standing monolayer $\mathrm{WTe}_{2}$ is grown on a supporting template. Even though we expect the bonding to be weak in absolute terms and of vdw nature, the lattice commensuration plays a crucial role as it induces strain. Bilayer graphene (BLG) is a typical substrate used to grow monolayers of transition metal dichalcogenides. In the specific case of $\mathrm{WTe}_{2}$, we find that a $2 \times 2$ reconstruction on BLG $3 \times 6$ induces a lateral tensile strain of $\sim 5.5 \%$ along the $\mathrm{W}$ zigzag chains. In Fig. 1 $1 \mathrm{p}$ we show the binding energy of $\mathrm{WTe}_{2}$ on BLG computed by explicitly including the vdw long range interactions. At the equilibrium distance $d_{\text {eq }}$, the vertical separation between BLG and the topmost Te layers of $\mathrm{WTe}_{2}$ is $\sim 7.5 \AA$, a value that fits well with the STM measurements reported in [31].

The band structure, when unfolded in the primitive Brillouin zone [29, 30], does not show the semimetallic character typical of free-standing $\mathrm{WTe}_{2}$, but instead is characterized by a positive indirect gap (Fig. $2 \mathrm{p}$ ). This result originates from the reduction of the hopping parameters along the $\mathrm{W}$ chains, an explicit consequence of the tensile strain [27]. Moreover, from the spin splitting of the electronic states around the gapped Dirac cone, we estimate that the strength of the inversion symmetry breaking is $\lesssim 10 \mathrm{meV}$. The small magnitude can be attributed to the weak vdw coupling to the substrate.

Effective tight-binding model - Based on the DFT band structure, we provide the minimal eight-band spin-orbit coupled tight-binding model that has the same spatial symmetries as monolayer $\mathrm{WTe}_{2}$ and quantitatively reproduces its low energy band structure in a window of about $1 \mathrm{eV}$ around the Fermi energy. The symmetries of free-standing monolayer $\mathrm{WTe}_{2}$ are time-reversal $T$, a glide mirror $\bar{M}_{x}$ that sends $x \mapsto-x$ combined with a half lattice translation in $x$ direction, and a two-fold screw symmetry around the $x$ axis $\bar{C}_{2 x}$ with the same translation as $\bar{M}_{x}$. The product of $\bar{M}_{x}$ and $\bar{C}_{2 x}$ is the three-dimensional inversion $\mathcal{I}$. The latter implies a twofold spin-degeneracy of all bands, and $\bar{M}_{x}$ implies that pairs of these two-fold degenerate bands join into a four-fold degeneracy at $k_{x}=\pi$. Thus, the minimal insulating band structure with these symmetries has eight bands. Building up on the results of [27], we choose the corresponding degrees of freedom as spin $s=\uparrow, \downarrow$, sublattice $\kappa=A, B$, and Wannier orbitals $\ell=p, d$. We let the Pauli matrices $\sigma_{\nu}, \rho_{\nu}$, and $\tau_{\nu}$, for $\nu=0,1,2,3$, act on the $s, \kappa$, and $\ell$ degree of freedom, respectively. (Here, $\nu=0$ labels the identity matrices.) The symmetries are then represented by $T=K \sigma_{2} \rho_{0} \tau_{0}$, mapping 
a)

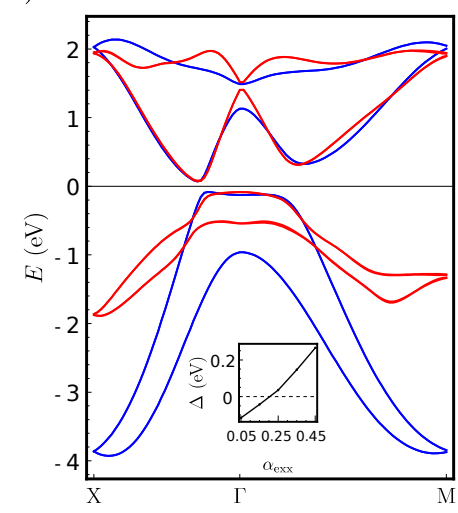

b)

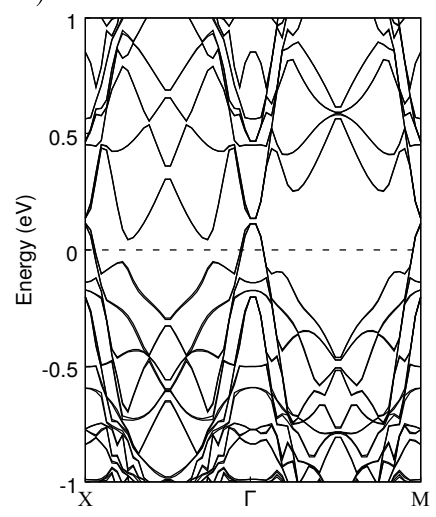

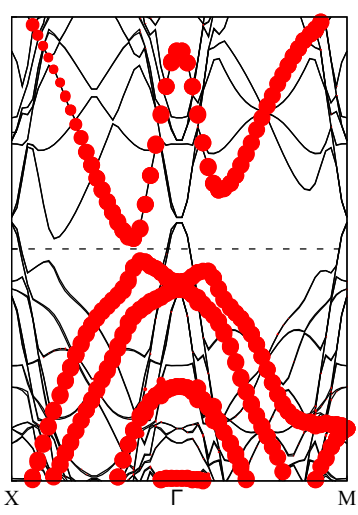

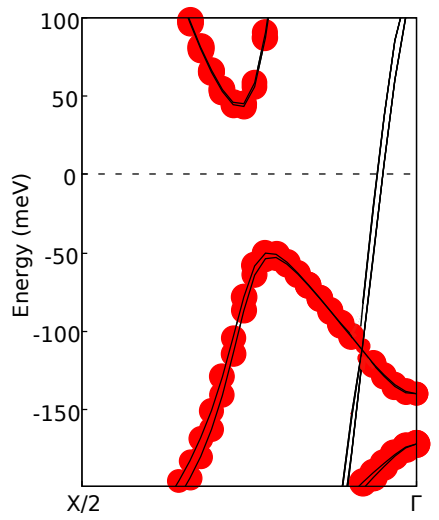

FIG. 2. a) Comparison of DFT HSE06 band structure (red) and tight-binding band structure (blue) including SOC. The tight-binding model (1) with SOC term of strength $V=0.115 \mathrm{eV}$ was used, while $\mathcal{H}_{\mathrm{R}}^{\mathrm{SOC}}$ was neglected. The inset shows the evolution of the indirect band gap induced by SOC as a function of the Hartree-Fock exchange. The red curve corresponds to the HSE06 value $\alpha_{\operatorname{exx}}=0.25$. b) Left: Band structure of monolayer $\mathrm{WTe}_{2}(2 \times 2$ unit cells $)$ on BLG $(3 \times 6$ unit cells $)$ along the high-symmetry lines of the unfolded primitive Brillouin zone. Middle: Same as left where the red circles highlight the unfolding coefficients originating from the unfolding procedure. These weights are defined from the scalar product $\langle K N \mid k n\rangle$, where upper (lower) case symbols $K$ and $N$ ( $k$ and $n$ ) identify supercell (primitive cell) momenta and band indices, and are interpreted as proper spectral weights that allow to efficiently map the band structure of the primitive cell out of the band structure of the supercell. A derivation of the unfolding strategy can be found in Refs. [28,-30]. Right: Zoom around the Dirac cone dispersion. The small splitting of the bands is due to Rashba SOC.

$\boldsymbol{k} \mapsto-\boldsymbol{k}, \bar{M}_{x}=\sigma_{1}\left[\rho_{0}\left(1+e^{\mathrm{i} k_{x}}\right)+\rho_{3}\left(1-e^{\mathrm{i} k_{x}}\right)\right] \tau_{0} / 2$, mapping $\left(k_{x}, k_{y}\right) \mapsto\left(-k_{x}, k_{y}\right)$, and $\bar{C}_{2 x}=\sigma_{1}\left[\rho_{1}\left(1+e^{\mathrm{i} k_{x}}\right)+\right.$ $\left.\mathrm{i} \rho_{2}\left(1-e^{\mathrm{i} k_{x}}\right)\right] \tau_{0} / 2$, mapping $\left(k_{x}, k_{y}\right) \mapsto\left(k_{x},-k_{y}\right)$. We write the tight-binding Hamiltonian directly in its Bloch representation in momentum space. Furthermore, we split the model into a spin-rotation invariant contribution without spin-orbit coupling, an intrinsic (int) SOC term which still preserves U(1) spin symmetry, and a Rashba (R) type contribution

$$
\mathcal{H}(\boldsymbol{k})=\mathcal{H}_{0}(\boldsymbol{k})+\mathcal{H}_{\mathrm{int}}^{\mathrm{SOC}}+\mathcal{H}_{\mathrm{R}}^{\mathrm{SOC}},
$$

with $\mathcal{H}_{0}(\boldsymbol{k}), \mathcal{H}_{\text {int }}^{\mathrm{SOC}}$, and $\mathcal{H}_{\mathrm{R}}^{\mathrm{SOC}}$ to be specified below. The explicit form of the Hamiltonian contribution without SOC is

$\mathcal{H}_{0}(\boldsymbol{k})=\sigma_{0} \otimes\left(\begin{array}{cccc}\epsilon_{d}(\boldsymbol{k}) & 0 & \tilde{t}_{d} g_{k_{x}} e^{\mathrm{i} k_{y}} & \tilde{t}_{0} f_{k_{x}} \\ 0 & \epsilon_{p}(\boldsymbol{k}) & -\tilde{t}_{0} f_{k_{x}} & \tilde{t}_{p} g_{k_{x}} \\ \tilde{t}_{d} g_{k_{x}}^{*} e^{-\mathrm{i} k_{y}} & -\tilde{t}_{0} f_{k_{x}}^{*} & \epsilon_{d}(\boldsymbol{k}) & 0 \\ \tilde{t}_{0} f_{k_{x}}^{*} & \tilde{t}_{p} g_{k_{x}}^{*} & 0 & \epsilon_{p}(\boldsymbol{k})\end{array}\right)$,

where $g_{k_{x}}=1+e^{-\mathrm{i} k_{x}}, f_{k_{x}}=1-e^{-\mathrm{i} k_{x}}$, and $\epsilon_{\ell}(\boldsymbol{k})=\mu_{\ell}+$ $2 t_{\ell} \cos k_{x}+2 t_{\ell}^{\prime} \cos 2 k_{x}$ for $\ell=p, d$. By choosing a set of parameters $\left(\mu_{d}=0.4935, \mu_{p}=1.3265, t_{d}=-0.28, t_{d}^{\prime}=\right.$ $0.075, t_{p}=0.93, t_{p}^{\prime}=0.075, t_{d}^{A B} 0.52, t_{p}^{A B}=0.40, t_{0}^{A B}=$ 1.02) in $\mathrm{eV}$ units, we get a dispersion which fits well with HSE06 result near the Fermi level.

In order to reproduce the gapped dispersion observed in both the case of a substrate or the calculations with HSE06, we include a spin-orbit coupling term that preserves i) TRS, ii) mirror symmetry, and iii) glide symmetry. Among a number of possibilities, we particularize to the ones at lowest order in $\boldsymbol{k}=\left(k_{x}, k_{y}\right)$, in line with our goal to find the simplest SOC terms. In the basis we chose in Eq. (2), there are two constant terms that we use in this paper: $\sigma_{2} \rho_{3} \tau_{2}$ and $\sigma_{3} \rho_{3} \tau_{2}$. Introduc- ing those, we obtain an intrinsic SOC term of the form

$$
\mathcal{H}_{\text {int }}^{\text {SOC }}=V \sigma_{2} \rho_{3} \tau_{2}+V^{\prime} \sigma_{3} \rho_{3} \tau_{2},
$$

with coefficients $V$ and $V^{\prime}$. One finds that those two types of terms are related by spin-rotation by $\pi / 4$ around $\sigma_{1}$, and that the spin-rotation also satisfies all symmetries, as it is a global unitary transformation of the Hamiltonian. Therefore, the two terms act equivalently in the Hamiltonian: The spectrum and the topology of the Hamiltonian with SOC, Eq. (3), is invariant as long as $V^{2}+V^{\prime 2}$ is kept unchanged. Unless otherwise noted, we assume $V^{\prime}=0$ throughout the rest of the paper. We checked that for $V=0.115 \mathrm{eV}$, the dispersion in a window of about $\pm 0.5 \mathrm{eV}$ around the Fermi level is very similar to the HSE06 and substrate calculations with SOC (Fig. 2a).

To include the effect of the substrate in the tight-binding model (1), we add Rashba-type SOC terms $\mathcal{H}_{\mathrm{R}}^{\mathrm{SOC}}$. We choose the ones with the lowest order (constant in $k_{x}$ and $k_{y}$ ) that preserve time-reversal and mirror symmetry, but break the screw symmetry (and thus also break inversion). By symmetry considerations similar to those used to derive the intrinsic SOC terms (3), we get $\sigma_{2} \rho_{0} \tau_{2}$ and $\sigma_{3} \rho_{0} \tau_{2}$ as candidate matrices for the perturbations to the tight-binding Hamiltonian. One notable feature is that the two candidates are also related by spin-rotation by $\pi / 4$ around $\sigma_{x}$, identically to the intrinsic SOC term. This fact leads us to a classification depending on whether the intrinsic SOC and the Rashba term are parallel or perpendicular in spin space: The Rashba term $\sigma_{2} \rho_{0} \tau_{2}$ is parallel in spin to (and thus commuting with) the first term in the intrinsic SOC term (3), while it is orthogonal (anticommuting) with the second term. For the other Rashba term $\sigma_{3} \rho_{0} \tau_{2}$ the situation is reversed: It anticommutes with $\sigma_{2} \rho_{3} \tau_{2}$ and commutes with $\sigma_{3} \rho_{3} \tau_{2}$. Furthermore, a Zeeman field $\boldsymbol{B}$ can be 
a)

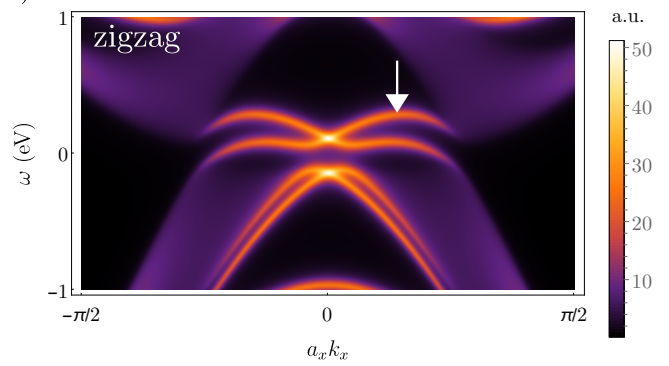

b)

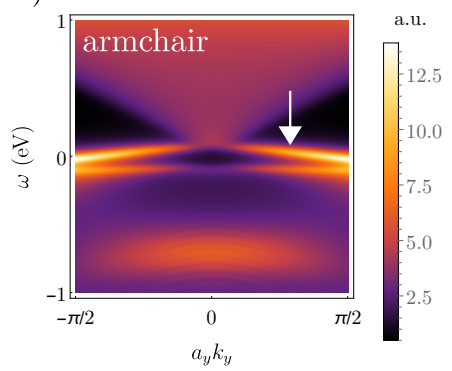

c)

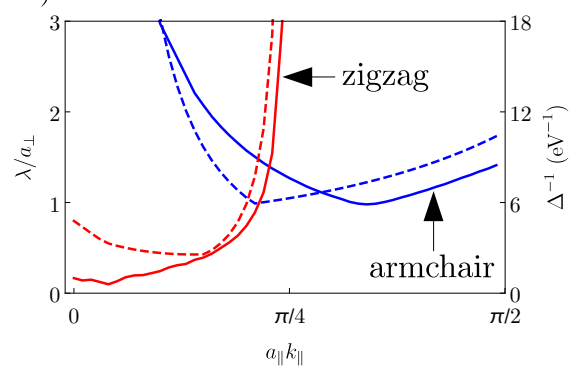

FIG. 3. a) Spectral function defined in Eq.4) showing the topological edge states of tight-binding model (1) on ribbon of 100 sites width with open boundary conditions in the $y$ direction. b) Same as a), but for open boundary conditions in the $x$ direction. c) Localization length (solid lines) and inverse gap (dashed lines) as a function of $k_{\|}=k_{x}$ for the case of a), and $k_{\|}=k_{y}$ for the case of b). The relevant bands are highlighted by white arrows in a) and b). Around $k_{x}=0$, the edge states are localized within a fraction of the unit cell due to the large direct bulk gap.

added to the tight-binding model (1) via the term $\boldsymbol{B} \cdot \boldsymbol{\sigma} \rho_{0} \tau_{0}$.

$Q S H$ edge modes - Using the value $V=0.115 \mathrm{eV}$ for the SOC amplitude in the following, we calculate the spectral functions in two different slab geometries

$$
A^{\lambda}\left(\omega, k_{\bar{\lambda}}\right)=\sum_{i} \operatorname{Im}\left[\frac{\left\langle\psi^{i}\left(k_{\bar{\lambda}}\right)\left|P_{\lambda}\right| \psi^{i}\left(k_{\bar{\alpha}}\right)\right\rangle}{\omega-E^{i}\left(k_{\bar{\lambda}}\right)+\mathrm{i} \delta}\right],
$$

with $\lambda=x, y(\bar{\lambda}=y, x$ for respective case) being the open (periodic) direction of the slab, $P_{\lambda}$ the one-edge projector for $\lambda$-slab, and we used $\delta=0.04$ for the Lorentzian broadening. The results are plotted in Fig. 3 and 3 p for the zig-zag and armchair edge, respectively. A stark difference between the two edges states is that the former has a Kramers degenerate point in the bulk gap, while the latter does not. Figure 3 ; shows the localization length of the edge modes as a function of the momentum parallel to the edge. We observe a particularly sharp localization of the order of one unit cell for the zigzag edge near the $k_{x}=0$ Kramers point. As expected, the localization length diverges at the momenta where the edge states connect to the bulk bands. In contrast, the localization length of the armchair edge states is much larger. For both edge orientations, the localization lengths correlate well with the inverse difference in energy between the edge state and the lowest bulk state (see dashed lines in Fig. 3k). This identifies the large direct bulk gap around the $\Gamma$ point as the origin of the extreme localization of the zigzag edge modes.

Furthermore, we investigate the response of the QSH edge modes to an external Zeeman field. As seen in [36], this magnetic response is highly sensitive to the type of termination and relative orientation of the Zeeman field. To leading order, the Zeeman effect opens a gap in the edge states if and only if the field points perpendicular to the spin-quantization axis singled out by the bulk spin-orbit coupling term $\left[\sigma_{2}\right.$ for the $V$ term and $\sigma_{3}$ for the $V^{\prime}$ term in Eq. [3] [34].

Discussion - Through developing an effective low-energy electronic model for monolayer $\mathrm{WTe}_{2}$, several directions of potential experimental and theoretical investigation offer themselves for further consideration. The motif to employ a glide symmetry to allow for Dirac cone gap opening away from high-symmetry points is likely to be applicable to a broad range of materials which so far have not yet been in the center of attention as candidates for quantum spin Hall effect. In the specific case of $\mathrm{WTe}_{2}$, substrate engineering might be intensified to optimize the electronic setting for a robust QSH phase. Furthermore, even though we in principle also find a consistent description for $\mathrm{WTe}_{2}$ without invoking strong electronic correlations, more sophisticated theoretical approaches may be used to analyze the role of electron-electron interactions in $\mathrm{WTe}_{2}$. Finally, the high sensitivity of the QSH edge mode localization length towards the specific termination can be probed in experiment. In this context, a rotation of the gate orientation might the most convenient way to pursue such an investigation.

R.T. thanks R. Cava and L. W. Molenkamp for discussions. The work was supported by ERC-StG-ThomaleTOPOLECTRICS-336012, ERC-StG-Neupert-757867PARATOP, DFG-SPP 1666, DFG-SFB 1170, "ToCoTronics", and the Swiss National Science Foundation (grant number: 200021_169061).

* rthomale@ physik.uni-wuerzburg.de

† titus.neupert@uzh.ch

[1] C. L. Kane and E. J. Mele, Physical Review Letters 95, 226801 (2005).

[2] B. A. Bernevig, T. L. Hughes, and S.-C. Zhang, Science 314, 1757 (2006).

[3] M. Z. Hasan and C. L. Kane, Reviews of Modern Physics 82, 3045 (2010).

[4] X.-L. Qi and S.-C. Zhang, Reviews of Modern Physics 83, 1057 (2011).

[5] M. König, S. Wiedmann, C. Brüne, A. Roth, H. Buhmann, L. W. Molenkamp, X.-L. Qi, and S.-C. Zhang, Science 318, 766 (2007).

[6] K. C. Nowack, E. M. Spanton, M. Baenninger, M. König, J. R. Kirtley, B. Kalisky, C. Ames, P. Leubner, C. Brüne, H. Buhmann, et al., Nature Materials 12, 787 (2013). 
[7] R. S. Deacon, J. Wiedenmann, E. Bocquillon, F. Domínguez, T. M. Klapwijk, P. Leubner, C. Brüne, E. M. Hankiewicz, S. Tarucha, K. Ishibashi, et al., Physical Review X 7, 021011 (2017).

[8] C. Liu, T. L. Hughes, X.-L. Qi, K. Wang, and S.-C. Zhang, Physical Review Letters 100, 236601 (2008).

[9] I. Knez, R.-R. Du, and G. Sullivan, Physical Review Letters 107, 136603 (2011).

[10] L. Du, I. Knez, G. Sullivan, and R.-R. Du, Physical Review Letters 114, 096802 (2015).

[11] F. Reis, G. Li, L. Dudy, M. Bauernfeind, S. Glass, W. Hanke, R. Thomale, J. Schäfer, and R. Claessen, Science 357, 287 (2017).

[12] G. Li, W. Hanke, E. M. Hankiewicz, F. Reis, J. Schäfer, R. Claessen, C. Wu, and R. Thomale, Physical Review B 98, $165146(2018)$

[13] S. Wu, V. Fatemi, Q. D. Gibson, K. Watanabe, T. Taniguchi, R. J. Cava, and P. Jarillo-Herrero, Science 359, 76 (2018).

[14] X. Qian, J. Liu, L. Fu, and J. Li, Science 346, 1344 (2014).

[15] M. N. Ali, J. Xiong, S. Flynn, J. Tao, Q. D. Gibson, L. M. Schoop, T. Liang, N. Haldolaarachchige, M. Hirschberger, N. Ong, et al., Nature 514, 205 (2014).

[16] A. A. Soluyanov, D. Gresch, Z. Wang, Q. Wu, M. Troyer, X. Dai, and B. A. Bernevig, Nature 527, 495 (2015).

[17] D. H. Keum, S. Cho, J. H. Kim, D.-H. Choe, H.-J. Sung, M. Kan, H. Kang, J.-Y. Hwang, S. W. Kim, H. Yang, et al., Nature Physics 11, 482 (2015).

[18] J. Jiang, F. Tang, X. Pan, H. Liu, X. Niu, Y. Wang, D. Xu, H. Yang, B. Xie, F. Song, et al., Physical Review Letters 115, 166601 (2015).

[19] D.-H. Choe, H.-J. Sung, and K. J. Chang, Physical Review B 93, 125109 (2016).

[20] F. Zheng, C. Cai, S. Ge, X. Zhang, X. Liu, H. Lu, Y. Zhang, J. Qiu, T. Taniguchi, K. Watanabe, et al., Advanced Materials 28, 4845 (2016).

[21] D. Di Sante, P. K. Das, C. Bigi, Z. Ergönenc, N. Gürtler, J. Krieger, T. Schmitt, M. Ali, G. Rossi, R. Thomale, et al., Physical Review Letters 119, 026403 (2017).

[22] Z. Fei, T. Palomaki, S. Wu, W. Zhao, X. Cai, B. Sun, P. Nguyen, J. Finney, X. Xu, and D. H. Cobden, Nature Physics 13, 677 (2017).

[23] V. Fatemi, S. Wu, Y. Cao, L. Bretheau, Q. D. Gibson, K. Watanabe, T. Taniguchi, R. J. Cava, and P. Jarillo-Herrero, Science 10, 1126 (2018).

[24] Z. Fei, W. Zhao, T. A. Palomaki, B. Sun, M. K. Miller, Z. Zhao, J. Yan, X. Xu, and D. H. Cobden, Nature 560, 336 (2018).

[25] P. K. Das, D. Di Sante, I. Vobornik, J. Fujii, T. Okuda, E. Bruyer, A. Gyenis, B. Feldman, J. Tao, R. Ciancio, et al., Nature Communications 7, 10847 (2016).

[26] Y. Wu, N. H. Jo, D. Mou, L. Huang, S. L. Bud'ko, P. C. Canfield, and A. Kaminski, Physical Review B 95, 195138 (2017).

[27] L. Muechler, A. Alexandradinata, T. Neupert, and R. Car, Physical Review X 6, 041069 (2016).

[28] V. Popescu and A. Zunger, Physical Review Letters 104, 236403 (2010).

[29] W. Ku, T. Berlijn, C.-C. Lee, et al., Physical Review Letters 104, 216401 (2010).

[30] M. Tomić, H. O. Jeschke, and R. Valentí, Physical Review B 90, 195121 (2014).

[31] S. Tang, C. Zhang, D. Wong, Z. Pedramrazi, H.-Z. Tsai, C. Jia, B. Moritz, M. Claassen, H. Ryu, S. Kahn, et al., Nature Physics 13, 683 (2017).

[32] Y.-H. Song, Z.-Y. Jia, D. Zhang, X.-Y. Zhu, Z.-Q. Shi, H. Wang, L. Zhu, Q.-Q. Yuan, H. Zhang, D.-Y. Xing, et al., Nature Com- munications 9, 4071 (2018)

[33] D. Rhodes, S. Das, Q. R. Zhang, B. Zeng, N. Pradhan, N. Kikugawa, E. Manousakis, and L. Balicas, Physical Review B 92, 125152 (2015).

[34] Refer to the Supplemental Material.

[35] N. Mardirossian and M. Head-Gordon, Molecular Physics 115, 2315 (2017).

[36] F. Dominguez, B. Scharf, G. Li, J. Schäfer, R. Claessen, W. Hanke, R. Thomale, and E. M. Hankiewicz, Physical Review B 98, 161407 (2018) 


\section{SUPPLEMENTARY INFORMATION}

Effect of Zeeman field on the edge states

a)

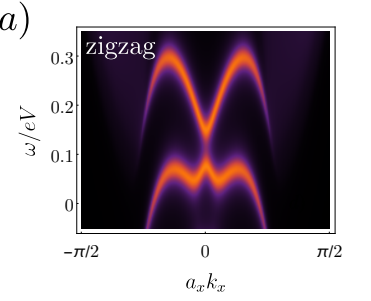

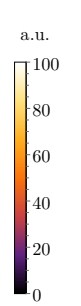

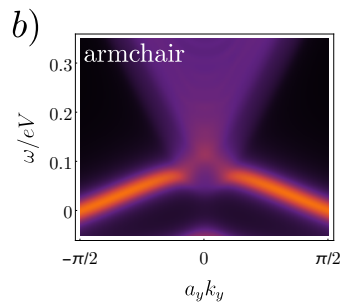

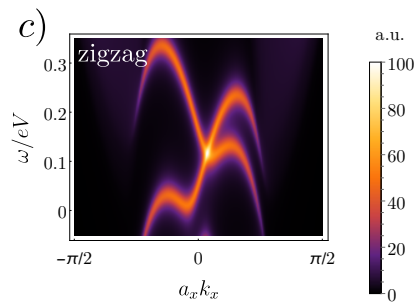

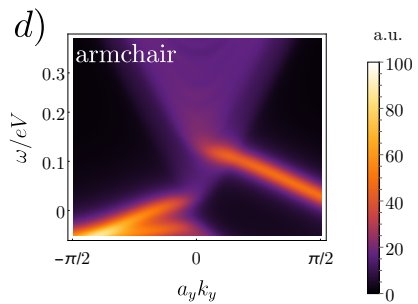

FIG. 4. Calculation results of spectral function (4) with the effect of a Zeeman field at the magnitude $0.05 \mathrm{eV}$. The Zeeman field is perpendicular (parallel) to the spin quantization axis defined by the intrinsic SOC in the left (right) two panels, resulting in the gap-opening (tilting) of the middle states. a) and c) is made in a ribbon geometry open in the $y$ direction, whereas b) and d) is open in the $x$ direction.

To study how a Zeeman field affects the topological edge states of $\mathrm{WTe}_{2}$, we study the Hamiltonian

$$
\widetilde{\mathcal{H}}(\boldsymbol{k})=\mathcal{H}_{0}(\boldsymbol{k})+\mathcal{H}_{\text {int }}^{\mathrm{SOC}}+\mathcal{H}^{\mathrm{Z}}
$$

with

$$
\mathcal{H}^{\mathrm{Z}}=B_{1} \sigma_{1} \rho_{0} \tau_{0}+B_{2} \sigma_{2} \rho_{0} \tau_{0}+B_{3} \sigma_{3} \rho_{0} \tau_{0}
$$

representing the Zeeman term. Similarly to the case of $\mathcal{H}_{\mathrm{R}}^{\mathrm{SOC}}, \mathcal{H}^{\mathrm{Z}}$ also splits into two components: the one parallel to the intrinsic SOC, and the other perpendicular to it. In Fig. 4, we present the numerical result of Eq. (4) using Hamiltonian Eq. (5) in both cases for two different edge terminations, i.e., zigzag and armchair. We observe that the perpendicular Zeeman field lifts the Dirac crossing of the topological boundary state, whereas the parallel one twists the edge states into an asymmetric form in $\boldsymbol{k}$-space, preserving the Dirac crossing. The gaplessness of the edge states in the latter case is protected by the (artificial) spin-conservation along the axis of the magnetic field.

\section{Comparison of GGA and HSE06 for the band structure calculation}

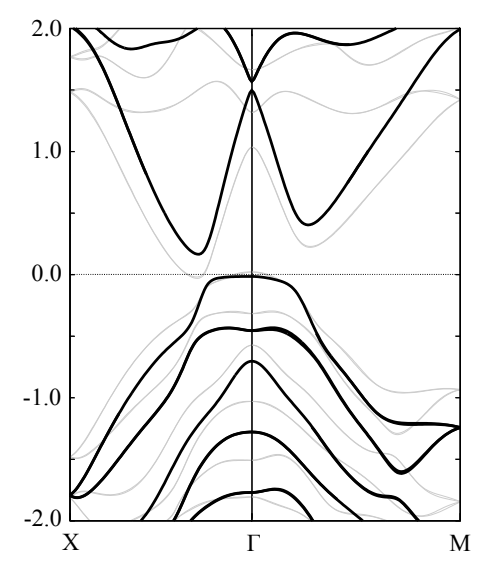

FIG. 5. Band structure of monolayer WTe 2 with SOC calculated using GGA (gray lines) and HSE06 (thick black lines) as exchange-correlation functionals.

In Fig. 5] we present the direct comparison between the band structures obtained from GGA and HSE06 with a Hartree-Fock exchange of $\alpha_{\mathrm{exx}}=0.25$. It is well known that GGA may over-emphasizes metallic screening effects due to an underestimate of band gaps. The inclusion of direct exchange mitigates this effect by reducing the self-interaction errors and leads to a band gap more in line with the experimental results. 